\title{
Strength and dynamic characteristics analyses of wound composite axial impeller
}

\author{
Jifeng Wang*, Jorge Olortegui-Yume, Norbert Müller \\ Turbomachinery Lab, Mechanical Engineering department, Michigan State University \\ East Lansing, MI 48824, USA
}

Received 3 April 2011 ; accepted 11 October 2011

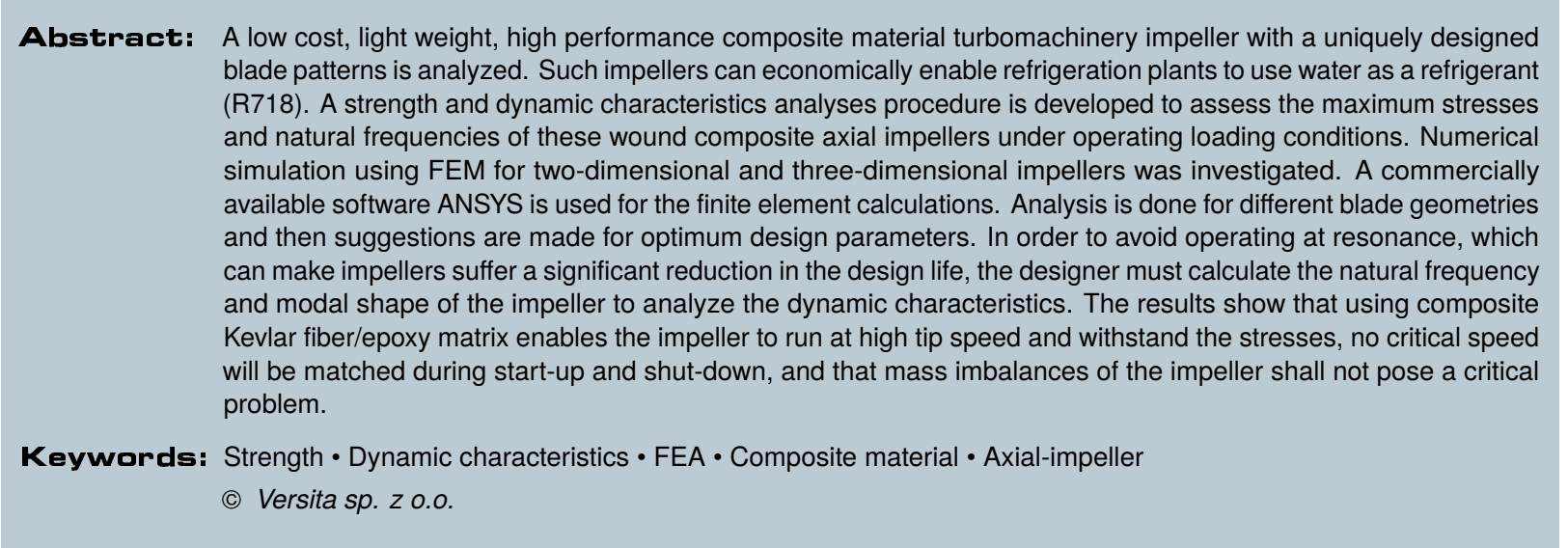

\section{Introduction}

A wound composite material axial impeller has been developed and is intended to be used for refrigeration purposes using water as a refrigerant (Fig. 1). The benefits of utilizing water as a refrigerant are widely known and vast [1] Water is one of the most natural refrigerants and it may be taken right from the tap. The utilization of water as a refrigerant can yield a very high coefficient of performance (COP) or energy efficiency rating (EER) [2-5]. Addition-

${ }^{*}$ E-mail: jwang94@illinois.edu ally, such technology has been shown low noise emissions even for large scale units. Due to very high latent and apparent heat properties, water can achieve an unusually high efficiency. Since the cycle works under vacuum, the volumetric cooling capacity of water vapor is very low and therefore huge volume flows need to be compressed with relatively high pressure ratios. This can only be achieved economically with customized high-performance turbo compressors.

Due to high pressure ratio involved, the tip speed for these impellers is also very high compared to impellers used to compress other fluids [6]. Working under vacuum, the forces exerted by the vapor on the blades are typically very small for the considered application [7], so in this particular case 


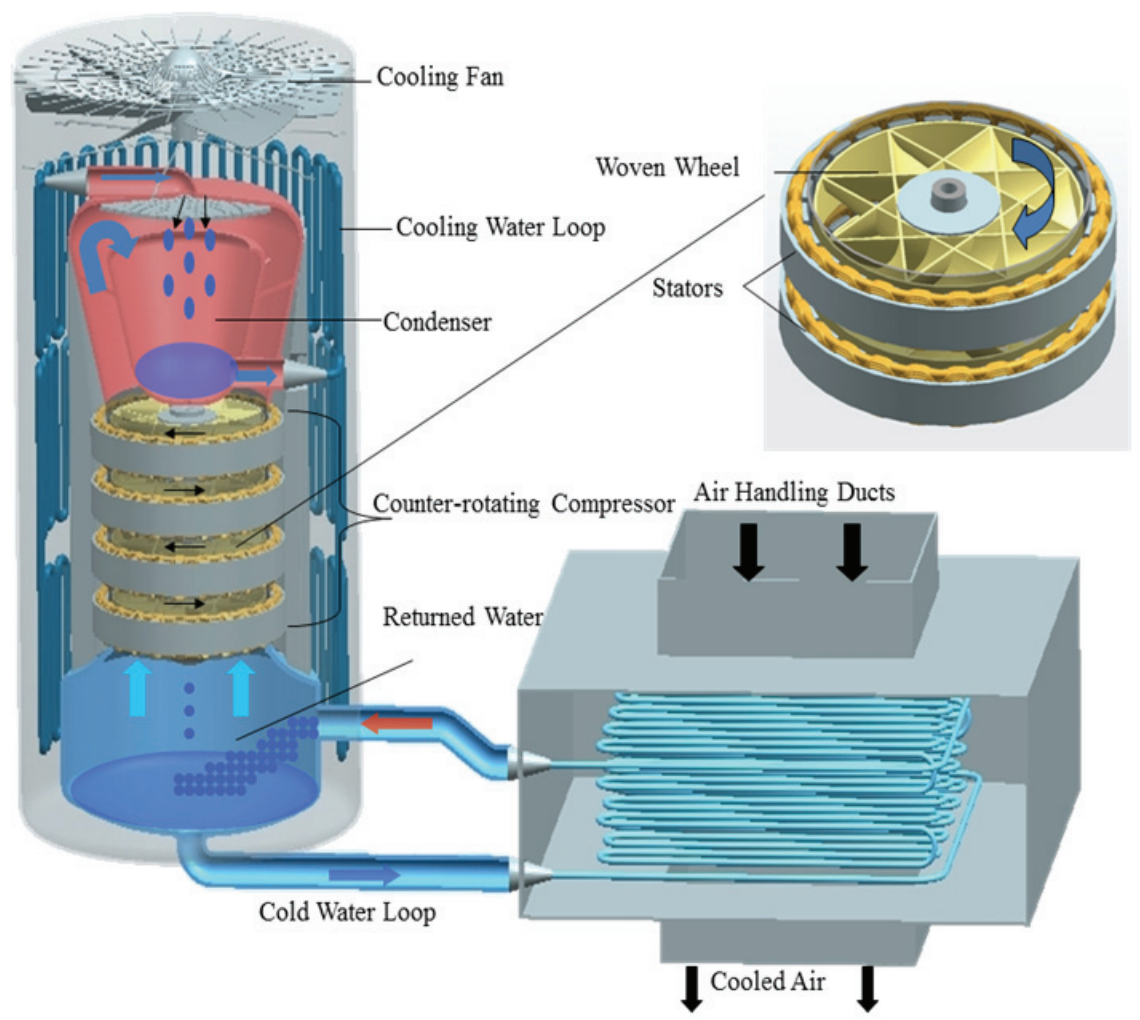

Figure 1. Chiller system using water as refrigerant.

the blades mainly have to withstand centrifugal forces, and the stress distribution is calculated predominantly due to them [8]. Therefore, the blades and their fixture must largely withstand centrifugal forces resulting from their inherent mass. So high-strength, light-weight structures are preferred, hence composites. GE developed the first turbofan jet GE90 with both fan case and fan blades made of composite materials, which provide significant weight reduction, reduced fuel consumption and enhanced engine durability. Because the structure was made of composite material the lighter blades permitted weight savings elsewhere in the engine. Reduced internal stresses within the fan assembly mean that it can be less highly engineered for strength and therefore lighter overall [9]. Ziehl-Abegg produced a new impeller using the specially developed high-performance composite material ZAmid, which can reduce weight of more than $50 \%$ as compared to a steel impeller and reach a higher tip speed [10]. Typically, impellers used for water chillers are made manually because of the huge size and complex shape, but Michigan State University's Turbomachinery Lab has developed a way to mass-produce these impellers using the concept of filament winding (Fig. 2). This paper analyzed different possible impeller design patterns for a turbo compressor based on a composite material winding technology.

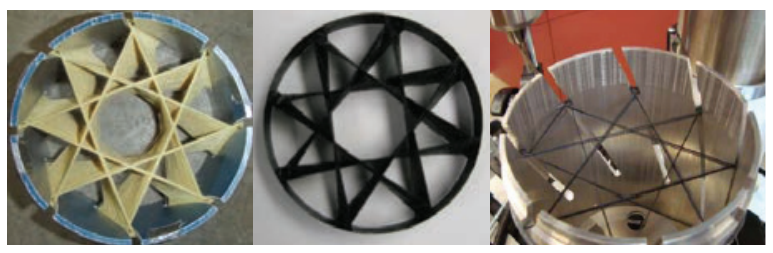

Figure 2. Rapid prototypes of wound impeller using winding machine.

The design of any turbomachinery impeller is a complex interdisciplinary process involving aerodynamics, thermodynamics, fluid dynamics, structural analysis, material selection, and requirements for manufacturing. Among these analyses, the one most frequently used in manufacturing is the structural analysis. Structural analysis is the process by which the relevant data for the structure are obtained. These data can be the following: stresses, deformations, displacements, oscillations, etc. It can be said that structural analysis presents an integral and important part of the design. Two types of methods for structural analysis: analytical and numerical usually can be combined and validated by experimental results [11]. In this study, 2-dimensional and 3-dimensional numerical strength and dynamic characteristics of some of the designed impellers 


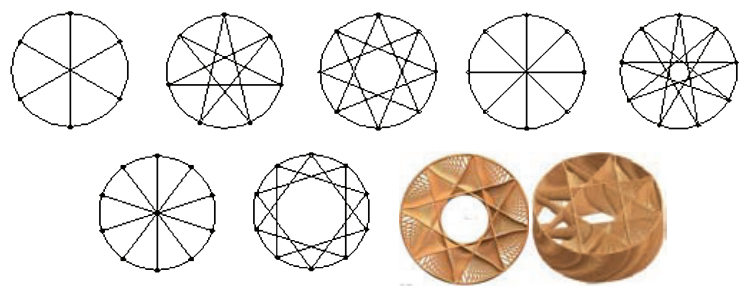

Figure 3. Different patterns of wound impellers.

patterns (Fig. 3) are investigated using Finite Element Methods (FEM) before the first prototype is built.

\section{Material characteristics}

\section{Nomenclature}

$E_{f} \quad$ Young's modulus of fiber, GPa

$E_{m} \quad$ Young's modulus of matrix, GPa

$E_{1} \quad$ Modulus in direction longitudinal to fiber, GPa

$E_{2} \quad$ Modulus in direction transverse to fiber, GPa

$G_{f} \quad$ Shear modulus of fiber, GPa

$G_{m} \quad$ Shear modulus of matrix, GPa

$G_{12}$ In-plane shear modulus of material, GPa

$V_{f} \quad$ Volume fraction of fiber

$V_{m} \quad$ Volume fraction of matrix

$v_{f} \quad$ Poisson ratio of fiber

$v_{m} \quad$ Poisson ratio of matrix

Kevlar fiber with epoxy matrix is chosen as the composite material since it has light weight and also high strength. The density of Kevlar is about half that of glass fiber and its specific strength is among the highest of currently available fibers. Kevlar also has excellent toughness, ductility, and impact resistance, unlike brittle glass or graphite fibers [12]. According to the micromechanics model of composite materials, the composite material properties are determined from:

$$
\begin{gathered}
E_{1}=E_{f 1} V_{f}+E_{m} V_{m} \\
E_{2}=\left(\frac{V_{f}}{E_{f 2}}+\frac{V_{m}}{E_{m}}\right)^{-1} \\
v_{12}=v_{f} V_{f}+v_{m} V_{m} \\
G_{12}=\left(\frac{V_{f}}{G_{f 12}}+\frac{V_{m}}{G_{m}}\right)^{-1},
\end{gathered}
$$

where the indices 1 and 2 are referred to the direction longitudinal and perpendicular to the fibers. The mechanical properties of Kevlar fiber/epoxy matrix composite material are listed in Table 1.
Table 1. Properties of Kevlar/Epoxy.

\begin{tabular}{ll}
\hline Fiber volume ratio, $V_{f}$ & 0.6 \\
Density, $\rho, \mathrm{kg} / \mathrm{m}^{3}$ & 1380 \\
Longitudinal modulus, $E_{1}, \mathrm{GPa}$ & 80 \\
Transverse modulus, $E_{2}, \mathrm{GPa}$ & 5.5 \\
In-plane shear modulus, $G_{12}, \mathrm{GPa}$ & 2.2 \\
Out-of- plane shear modulus, $\mathrm{G}_{23}, \mathrm{GPa}$ & 1.8 \\
Major Poisson's ratio, $v_{12}$ & 0.34 \\
Out-of- plane Poisson's ratio, $v_{23}$ & 0.4 \\
Longitudinal tensile strength, $\sigma_{1}^{T}, \mathrm{GPa}$ & 1.4 \\
Longitudinal compressive strength, $\sigma_{1}^{C}, \mathrm{GPa}$ & 0.335 \\
Transverse tensile strength, $\sigma_{2}^{T}, \mathrm{GPa}$ & 0.03 \\
Transverse compressive strength, $\sigma_{2}^{C}, \mathrm{GPa}$ & 0.158 \\
In-plane shear strength, $t_{12}^{u l t}, \mathrm{GPa}$ & 0.049 \\
\hline
\end{tabular}

Preventing failure of composite material systems has been an important issue in engineering design. The two types of physical failures that occur in laminated composite structures are intralaminar and interlaminar failures. Since both failures in a laminated composite structure may reduce the structural stiffness and strength, the possibility that intralaminar and interlaminar failures occur in structural components is considered a design limit [9]. Strength is an inherent property of a mechanical element and is a characteristic of the material. To avoid impeller failure the design engineer must have positive assurance that stresses generated will never exceed the strength [13].

\section{Stress analysis}

Stress analysis is complex and highly repeated routine work. While the finite element analysis processed by ANSYS produces a large amount of output (including displacement and stress); the use of ANSYS can display and inspect the analysis results effectively. FEM is used to find out stresses and displacements in complex and unusually shaped components. There are linear and nonlinear FEM analyses. Linear FEM analysis is based on basic assumptions like theory of small deformation and material is linear elastic. A composite material is obviously heterogeneous at the constituent material level, with properties possibly changing from point to point [11]. In this study, it is assumed that the composite material Kevlar fiber/epoxy matrix is transversely isotropic and the stressstrain relationship for the material is linear elastic for small deformations.

The Following steps are carried out for the stress analysis of impeller in this study:

1. Formulation and solving of geometrical equations to obtain the coordinates on the impellers circumference. 
2. Utilizing MATLAB programming to generate the various geometries of the impeller patterns

3. Reading the geometry through ANSYS log files to generate uniquely designed patterns of 2-D models.

4. Generating 3-D models using NX (UNIGRAPHICS)

5. Stress analysis of 2-D and 3-D models and comparison of different patterns.

The stresses were calculated at finite locations using the finite element analysis based on the displacement method. The force equilibrium equation is:

$$
[K]\{U\}=\{F\}
$$

where $K$ is the rigidity matrix, $U$ are the node displacements and $F$ is the external applied force on a node. The displacements $U$ found by solving equation (5) are then used to calculate all stresses and strains [14]. The Governing Equations in cylindrical coordinate system are:

$$
\begin{array}{r}
\frac{\partial \sigma_{r}}{\partial r}+\frac{1}{r} \frac{\partial \tau_{r \theta}}{\partial \theta}+\frac{\partial \tau_{r z}}{\partial z}+\frac{1}{r}\left(\sigma_{r}-\sigma_{\theta}\right)+F_{r}=0 \\
\frac{\partial \tau_{r \theta}}{\partial r}+\frac{1}{r} \frac{\partial \sigma_{\theta}}{\partial \theta}+\frac{\partial \tau_{\theta z}}{\partial z}+\frac{2}{r} \tau_{r \theta}+F_{\theta}=0 \\
\frac{\partial \tau_{r z}}{\partial r}+\frac{1}{r} \frac{\partial \tau_{\theta z}}{\partial \theta}+\frac{\partial \sigma_{z}}{\partial z}+\frac{1}{r} \tau_{r z}+F_{z}=0
\end{array}
$$

where $F_{r}=\rho \omega^{2} r$ ( $\omega$ is the rotating speed, $\rho$ is the density), $F_{\theta}=0, F_{z}=\rho g$ ( $g$ is the gravity acceleration).

\subsection{Flywheel patterns}

Among these different patterns of wound impellers shown in Fig. 3, flywheel pattern is an important pattern due to its simple geometry and easily manufacturing capability. For the stress analysis of these impellers, a state of plane stress is assumed in this study. The material is modeled as Linear Orthotropic with same properties in the direction of perpendicular to the fibers. The 2-D analysis has been investigated in three different patterns:

- Six symmetrical spokes (impeller with six blades and an outer shroud)

- Eight symmetrical spokes (impeller with eight blades and an outer shroud)

- Ten symmetrical spokes (impeller with ten blades and an outer shroud)

The dimensions shown in Fig. 4 were chosen as per the design requirements and scale-down impeller sizes.

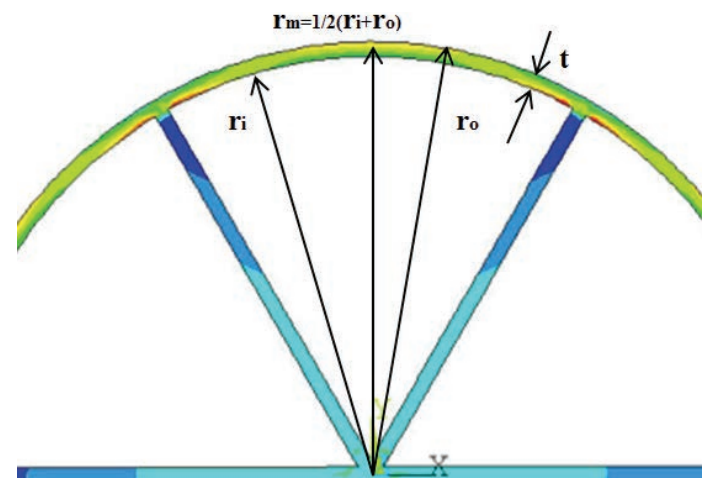

Figure 4. Schematic of parameters used in the impeller.

- Inner radius, $r_{i}=29 \mathrm{~mm}$

- Outer radius, $r_{o}=30 \mathrm{~mm}$

- Thickness of shroud and blade, $t=1 \mathrm{~mm}$

In this study, it is used Tsai-Wu failure criterion, which is one of the most used failure criteria for composite materials. For only one layer and plane stress state the Tsai-Wu criterion is simplified.

$$
\begin{aligned}
\sigma_{1}\left[\frac{1}{\left(\sigma_{1}^{T}\right)_{u l t}}-\frac{1}{\left(\sigma_{1}^{c}\right)_{u l t}}\right]+\sigma_{2}\left[\frac{1}{\left(\sigma_{2}^{T}\right)_{u l t}}-\frac{1}{\left(\sigma_{2}^{c}\right)_{u l t}}\right]+ & \frac{\sigma_{1}^{2}}{\left(\sigma_{1}^{T}\right)_{u l t}\left(\sigma_{1}^{c}\right)_{u l t}}+\frac{\sigma_{2}^{2}}{\left(\sigma_{2}^{T}\right)_{u l t}\left(\sigma_{2}^{c}\right)_{u l t}}+\frac{\tau_{12}^{2}}{\left(\tau_{12}\right)_{u l t}^{2}} \\
& -\frac{1}{2} \sigma_{1} \sigma_{2} \sqrt{\frac{1}{\left(\sigma_{1}^{T}\right)_{u l t}\left(\sigma_{1}^{c}\right)_{u l t}\left(\sigma_{2}^{T}\right)_{u l t}\left(\sigma_{2}^{c}\right)_{u l t}}}>1
\end{aligned}
$$

The state of stress values from ANSYS were fed into a MATLAB code that used equation (7) to check for failure.
Table 2 shows the Maximum tip speeds that different impeller patterns can attain just before failure. 
Table 2. Maximum tip speed for different impeller patterns.

\begin{tabular}{lcccc}
\hline & $\begin{array}{c}\text { First principal } \\
\text { stress (MPa) }\end{array}$ & $\begin{array}{c}\text { Second principal } \\
\text { stress (MPa) }\end{array}$ & $\begin{array}{c}\text { Plane shear } \\
\text { stress (MPa) }\end{array}$ & $\begin{array}{c}\text { Maximum tip } \\
\text { speed (m/s) }\end{array}$ \\
\hline \hline 6 blade flywheel impeller & 96.6 & 29.2 & 39.5 & 210 \\
8 blade flywheel impeller & 96.7 & 29.1 & 48.2 & 204 \\
10 blade flywheel impeller & 85.4 & 29.8 & 34.6 & 180 \\
\hline
\end{tabular}

Fig. 5 shows the Von-Misses stress distribution from ANSYS for the three patterns at a tip speed of $v_{o}=100 \mathrm{~m} / \mathrm{s}$, which lies in the desired operating speed range.

The 2-D analysis is further extended for the same tip speed of $100 \mathrm{~m} / \mathrm{s}$ and maximum stresses are found out at different mean radius to thickness $r_{m} / t$ ratios (Fig. 6). The analysis reveals that when tip speed is kept constant at the mean radius, the maximum stresses only depend on the ratio of mean radius to blade thickness (the same thickness with outer shroud) i.e. $r_{m} / t$. However, the relationship between the maximum stress and ratio $r_{m} / t$ is not linear, as shown in Fig. 6, and a peak of the maximum stresses exists for every pattern of impellers, which should be avoided in the design of impellers. It is observed that, when the $r_{m} / t$ ratio continues to increase, the maximum stress decreases and eventually becomes convergent upon a minimum value. This minimum value becomes the maximum stress for the outer shroud only with the same geometric parameter. Thus, due to the reduction in stress levels, the impeller should have a higher $r_{m} / t$ ratio.

\subsection{Three-Dimensional FEM stress analysis}

Similar to the 2-D case, the material is also modeled as Linear Orthotropic for 3-D study. For the 3-D analysis two different impeller patterns with eight blades were investigated, since eight blades impellers provide higher compression capability based on the Computational Fluid Dynamics (CFD) simulation. Fig. 7 shows three-dimensional FEM models for 8 blade flywheel impeller (named 8A) and another impeller pattern which also has the same 8 points on the impeller outer shroud (named $8 \mathrm{~B}$ ) in this study. The calculated radius ratio for $8 \mathrm{~B}$ of inner circle to outer circle is 0.383 . The geometry for 3-D FEM model was shown in Table 3.

Table 3. Geometry for 3-D FEM model.

\begin{tabular}{lc}
\hline Inner radius & $49 \mathrm{~mm}$ \\
Outer radius & $50 \mathrm{~mm}$ \\
Thickness of shroud and blade & $1 \mathrm{~mm}$ \\
Blade angle & $30^{\circ}$ \\
Impeller width & $38 \mathrm{~mm}$ \\
Tip speed & $100 \mathrm{~m} / \mathrm{s}$ \\
\hline
\end{tabular}

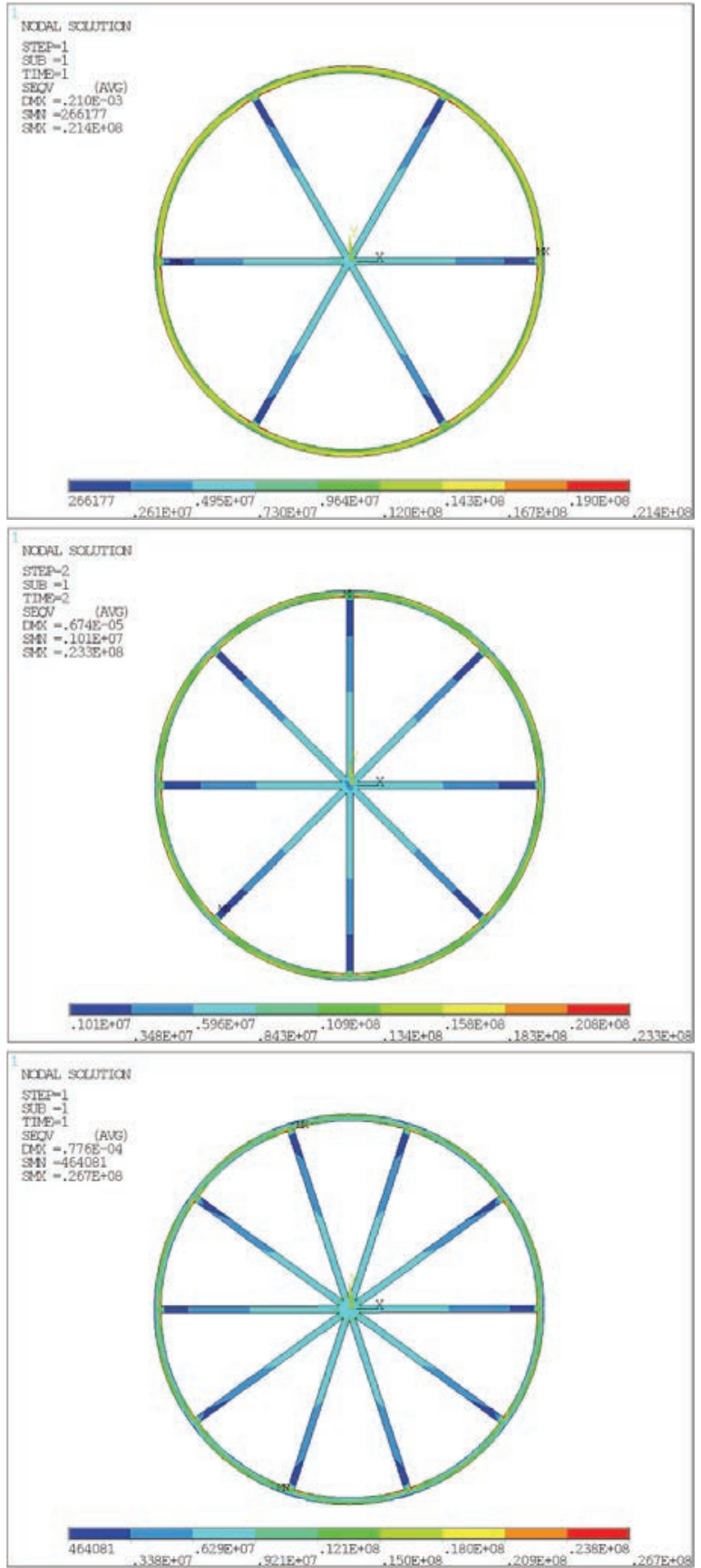

Figure 5. Plane stress distribution for flywheel pattern impellers. 


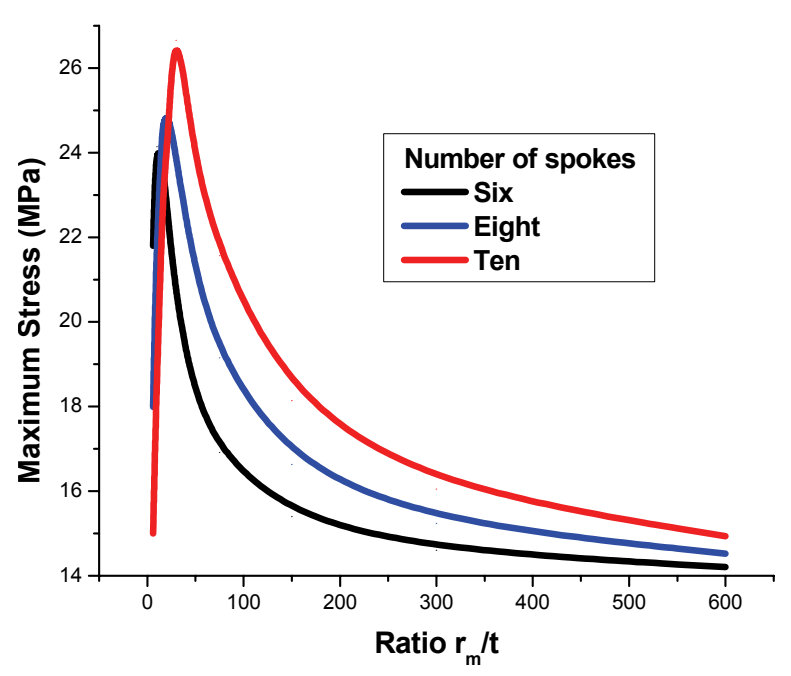

Figure 6. Maximum stress against ratio $r_{m} / t$ for three flywheel impellers.

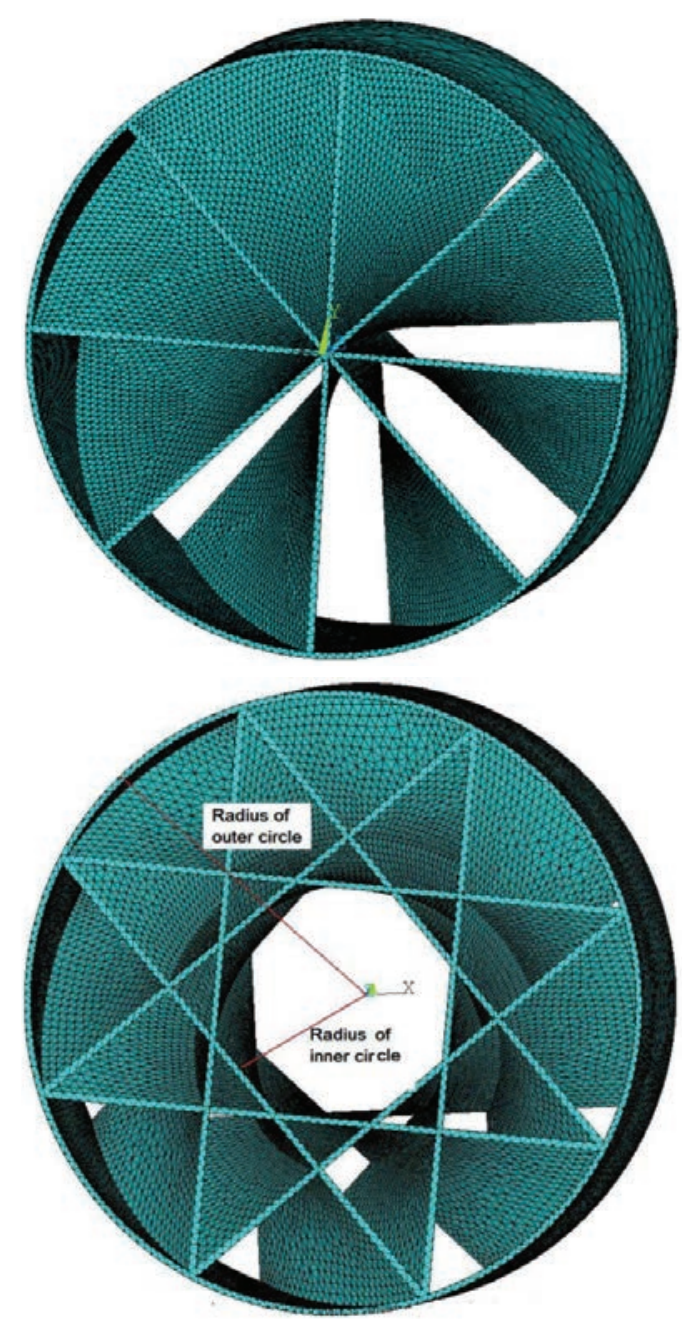

Figure 7. Finite element model of $8 \mathrm{~A}$ and $8 \mathrm{~B}$ impellers.

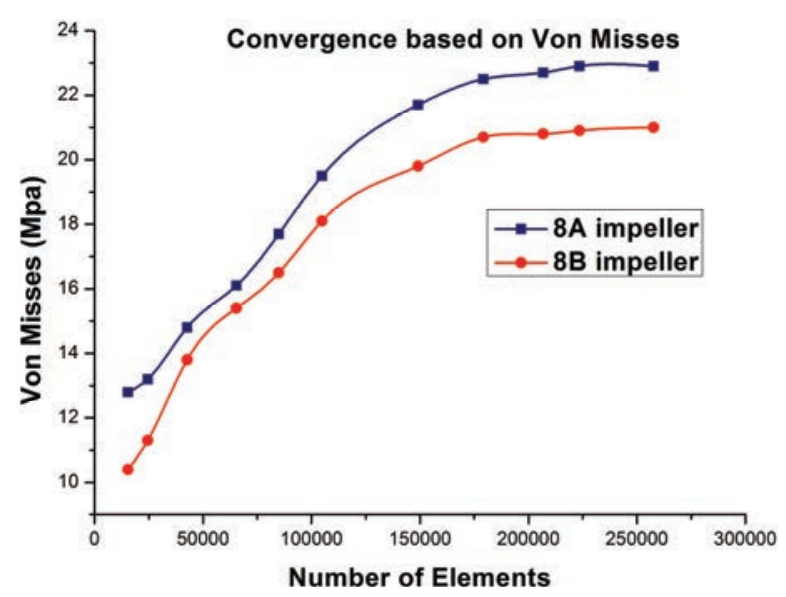

Figure 8. Convergence graph for 3-D FE model.

Nodes and elements can be generated by one of the three methods, free mesh, mapped mesh and sweep mesh. Here the free mesh is used for meshing because of more flexibility in defining mesh areas. In addition, it can be automatically created by an algorithm, which tries to minimize elements distortion. Convergence criteria should be considered to evaluate the results [15]. Convergence analysis is performed on the FE model. By increasing the mesh density in a step-by-step manner, a suitable number of elements is obtained. The stabilization of von Misses is considered for the criteria of convergence. Fig. 8 shows the results of convergence analysis. From Fig. 8, it is clear that convergence is obtained with the use of about 200,000 or more elements.

The results of maximum tangential, longitudinal, radial and shear stress are given by three-dimensional FEA [16]. All three principal stresses and shear stress acting on an impeller are calculated. Hence the Von Misses stress (also known as equivalent stress) with three principal stresses is given by Eq. (8) and its distribution is shown in Fig. 9 and Fig. 10.

$$
\sigma_{e q v}=\sqrt{\frac{\left(\sigma_{1}-\sigma_{2}\right)^{2}+\left(\sigma_{2}-\sigma_{3}\right)^{2}+\left(\sigma_{3}-\sigma_{1}\right)^{2}}{2}}
$$

Maximum Stress Failure Criterion predicts failure when any principal axis stress or the in plane shear stress component exceeds the corresponding strength. Thus, in order to avoid failure according to this criterion, the following set of inequalities must be satisfied:

$$
\begin{aligned}
& -\sigma_{1}^{C}<\sigma_{1}<\sigma_{1}^{T} \\
& -\sigma_{2}^{C}<\sigma_{2}<\sigma_{2}^{T} \\
& -\tau_{12 u l t}<\tau_{12}<\tau_{12 u l t}
\end{aligned}
$$




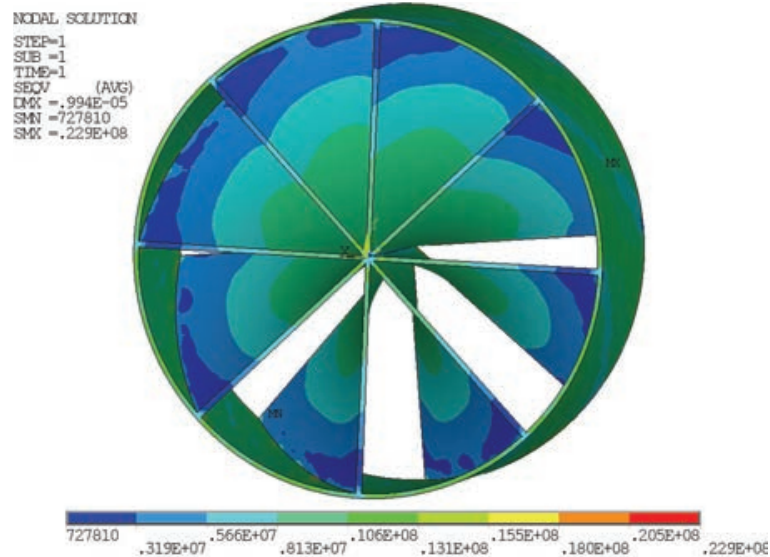

Figure 9. Von Misses distribution of 8A impeller.

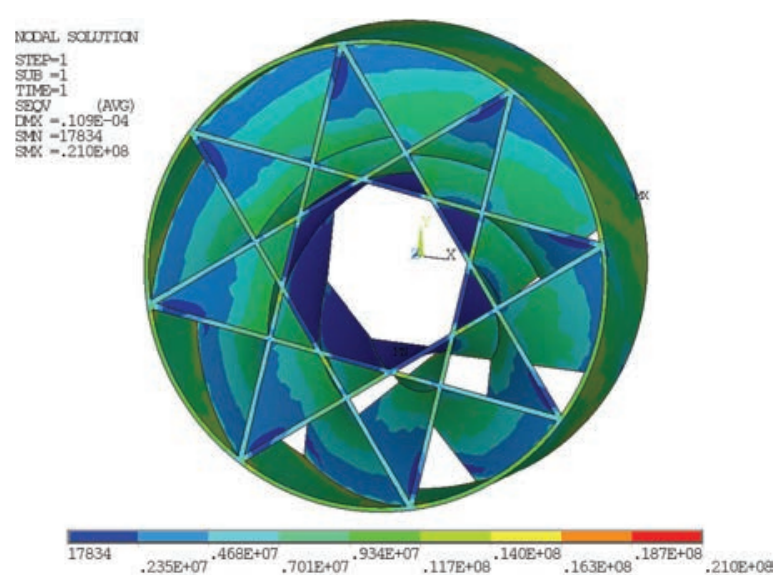

Figure 10. Von Misses distribution of $8 \mathrm{~B}$ impeller.

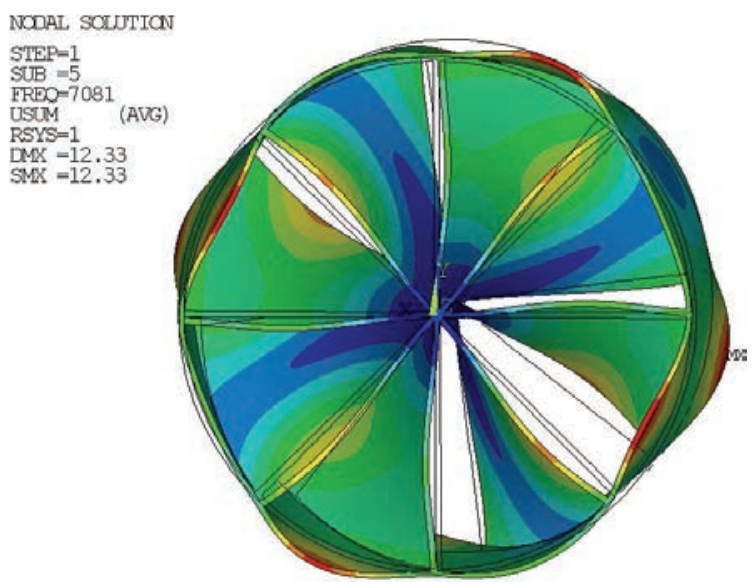

Figure 11. Fifth natural frequency and modal shape of $8 \mathrm{~A}$ impeller.
For the given set of parameters the impeller stresses were tested as per Eq. (9) and failure was not observed. It is observed that (in Fig. 9), the maximum stresses for the $8 \mathrm{~A}$ impeller (22.9 MPa) and the $8 \mathrm{~B}$ impeller (21 MPa) occur at the same place, inside the shroud. The magnitude of the stress for $8 \mathrm{~B}$ impeller is smaller than for that of the $8 \mathrm{~A}$ impeller. However, the locations of minimum stresses for these impellers are different. The minimum stress occurs on the tip of the blades for the $8 \mathrm{~A}$ impeller and at the root of the blades for the $8 \mathrm{~B}$ impeller, showing a wide range of variation. With the chosen high-strength Kevlar/epoxy composite structure, these wound impellers can withstand the required tip speeds tested here at $100 \mathrm{~m} / \mathrm{s}$.

\section{Dynamic Characteristics Analysis}

High-speed impellers can fail due to fatigue caused by vibration especially at resonance, a condition wherein the excitation frequency equals one of the natural frequencies of the system inducing very large deformations. Rotordynamics, a branch of dynamics applied to Turbomachinery, differentiate between static and dynamic natural frequencies which occur at specific geometric vibrational shapes named modal shapes. The centrifugal force influences the stiffness of the impeller which in turn changes the natural frequencies and modal shapes into their dynamics counterparts. The latter are functions of mass, mass distribution and speed which is why a vibration analysis of high-speed impellers has become essential to obtain higher tip speeds while reducing weight. The main purpose of the system's dynamics characteristics analysis is to determine the dynamic natural frequencies as well the modal shapes along with their impact over a range of tip speeds. In this analysis the impeller's natural frequencies and modal shapes must be obtained before going into the dynamic part. Accordingly, in this paper, modal analysis was done for the first ten modes assuming no external forces. The fifth natural frequency with mode shapes (deformed and un-deformed) for the $8 \mathrm{~A}$ and the $8 \mathrm{~B}$ impeller calculated are shown in Fig. 11 and Fig. 12.

Fig. 13 shows the first 10 undamped natural frequencies (static and dynamic) of the 8A impeller against the spin frequency. Fig. 13 is known as Campbell's diagram as long as synchronous lines, e.g. 1x-synchronous line, and tentative ranges of operation are considered, e.g. 15,000 and $25,000 \mathrm{rpm}$. From the plot it is observed that this impeller is operating sub-critically but very close to the 1 st natural frequency line $(1 \mathrm{M})$. In the actual implementation of the system some damping from the bearings should be included, resulting in lower damped natural frequencies. Thus, one crossing between the $1 \mathrm{X}$-Synchronous line and $1 \mathrm{M}$ is expected generating a point whose correspon- 


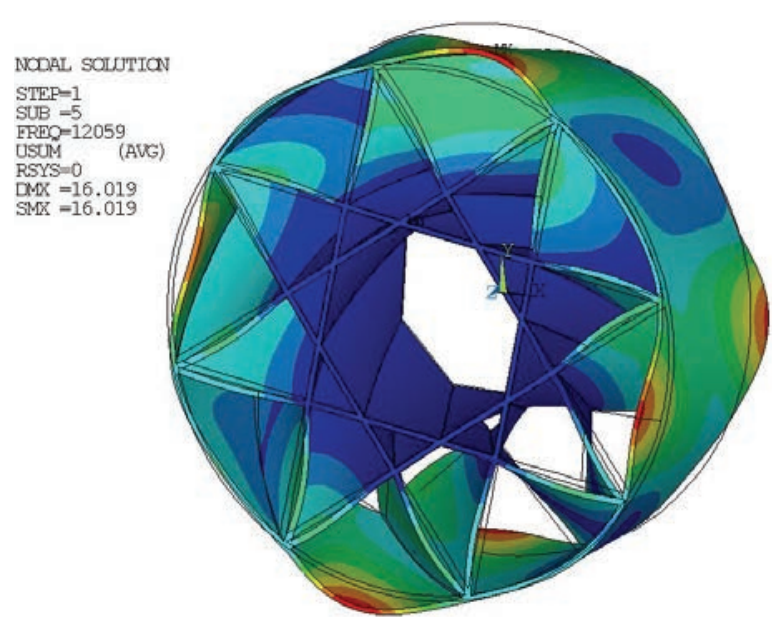

Figure 12. Fifth natural frequency and modal shape of 8B impeller.

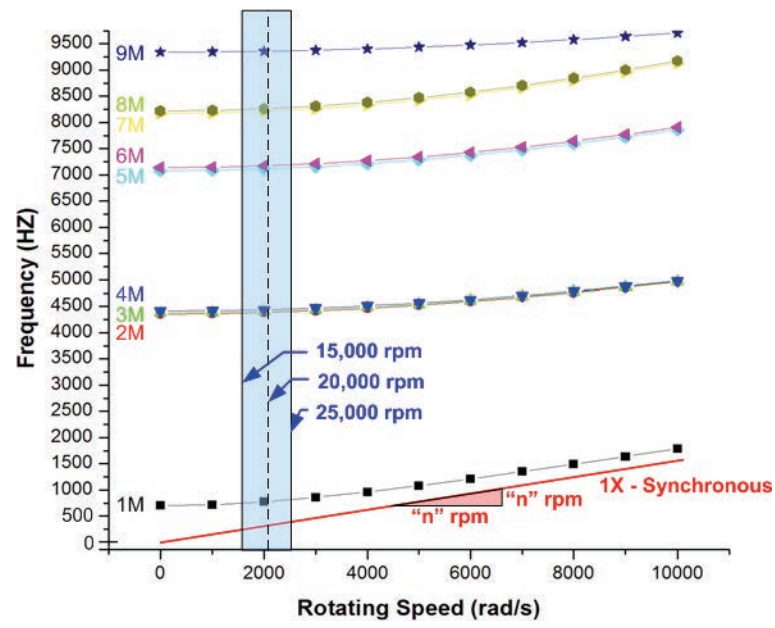

Figure 13. Campbell diagram of $8 \mathrm{~A}$ impeller.

dent speed value is called critical speed. In conclusion, adequate damping would be needed for the machine to go smoothly through this critical speed and sub-critical operation cannot be guaranteed.

The plot of the first 10 natural frequencies for the $8 \mathrm{~B}$ impeller versus spin frequency is shown in Fig. 14. It is observed that the impeller is operating sub-critically. This is an encouraging finding which means that an impeller's pattern change makes possible the avoidance of critical speeds associated with rotating unbalance. Plainly, rotating unbalance would not be dangerous at all as it will not be able to excite any natural frequencies of the impeller with the suitable impeller pattern. In the real case, the addition of damping will reduce the value of the original undamped natural frequencies; however, the damped natural frequencies are not expected to be reduced much compared

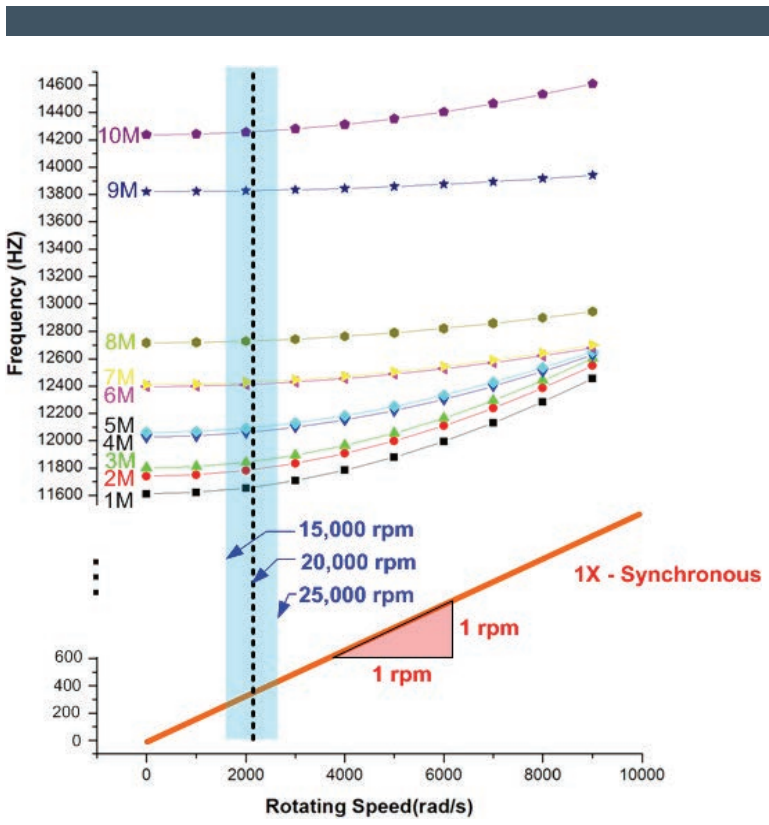

Figure 14. Campbell diagram of $8 \mathrm{~B}$ impeller.

to the undamped case, as damping for turbomachinery is known to be very low, i.e. damping ratio $(\xi)$ is usually less than 0.1 [17]. Therefore, sub-critical operation seems to be guaranteed.

\section{Conclusion}

In this paper different impeller patterns were analyzed for stress distribution and dynamic characteristics. 2-D FEA results for flywheel impellers with different number of blades were calculated. It was found out that the maximum stresses for flywheel impellers depends on the ratio of mean radius to thickness $\mathrm{rm} / \mathrm{t}$ and there is a particular ratio for each pattern which has a maximum stress (Fig. 5) which should be avoided by designers. 3-D FEM models were developed for two different 8 blade impellers and results were compared for chosen dimensions and operating conditions. It was found out that the $8 \mathrm{~B}$ impeller design is superior to the $8 \mathrm{~A}$ design because: (i) It had lower maximum stress for the same tip speed and same dimensions; (ii) 8B impeller has higher natural frequency than that of the $8 \mathrm{~A}$ impeller at the same rotating speed, which implies that the rotating unbalance will not represent a problem and cannot excite any undamped natural frequencies. It was also observed that the maximum stresses occur at the same location in both the impeller patterns.

Further development steps will include structural analysis of more impeller patterns. Computational Fluid Dynamics (CFD) simulations will also be used in conjunction with 
the stress distribution results from FEA (Fluid-Structure Interaction analysis) to further improve the impeller blade design. These steps will ensure that less time and resources are spent on fabricating and testing of impeller specimens and the final prototype will need little or no optimization.

\section{References}

[1] A. Kharazi, N. Müller, Comparing Water (R718) to Other Refrigerants, ASME-Paper IMECE2006-13341

[2] S. Wight, T. Yoshinaka, B. Le Drew and C. D'Orsi N, The efficiency limits of water vapor compressors, 2000 ARTI-21CR/605-10010-01

[3] B. Lachner, G.Nellis, and D. Reindl, The Use of Water Vapor as a Refrigerant Impact, 2004 ARTI-21CR/61110080-01

[4] BINE Informationsdienst, Germany, 2003, Wasser als Kältemittel (water as a refrigerant), project info 08/03, http://www.bine.info/pdf/publikation/ bi0803internetx.pdf

[5] A. Kharazi, P. Akbari and N. Müller, Analysis and Performance Prediction of an R-718 Compression $\mathrm{Cy}$ cle with a 3-Port Condensing Wave Rotor, J. Energ. Resour. Tech., 2006, Vol. 128, pp. 325-334

[6] N. Müller, 2001 Design of compressor impellers for water as a refrigerant ASHRAE Transactions, Vol. 107, pp. 214-222

[7] D. Bhope, P. Padole, Experimental and theoretical analysis of stresses, noise and flow in centrifugal fan impeller, Mech. Mach. Theor., 39(2004), 1257-1271
[8] A. Lahiri, B. Lindberg, N. Müller, Preliminary Mechanical Analysis for Novel Woven/Wound composite Axial-Compressor, ASME paper IMECE2007-42490

[9] P. Griggs, M.H. Schneider,GE90 fan blade service experience and repair, FAA/EASA/Industry Composite Damage Tolerance and Maintenance Workshop, 2007

[10] http://www . ziehl-abegg.com/ww/ news-711-Cpro---The-impeller-of-extremes . html

[11] D. Kreculj, Stress Analysis in an Unidirectional Carbon/Epoxy Composite Material, FMETransactions Vol. 36, No 3, 2008, pp. 127-132

[12] R. Gibson, Principles of composite material mechanics, McGraw Hill, 1994

[13] J.M.M. Asif, Optimization of location and size of opening in a pressure vessel cylinder using ANSYS. Eng. Fail. Anal., 2008; 15(1-2):1-19

[14] Z. Wang, Y. Luo, L. Zhou, R. Xiao and G. Peng. Computation of dynamic stresses in piston rods caused by unsteady hydraulic loads. Eng. Fail. Anal., 2008; 15(1-2): 28-37

[15] M. Shokrieh, R. Rafiee, Simulaiton of fatigue failure in a full composite wind turbine blade, Compos. Struct., 74(2006) 332-342

[16] J. Wang, M. Patil, J. Olortegui-Yume, N. Müller, Mechanical design of wound composite impeller using FEM, ASME paper IMECE2010-39762

[17] M. Adams, Rotating Machinery Vibration-From Analysis to Troubleshooting, Marcel Dekker, 2000 